low with no mortality within 28-days. Post-drain removal instructions and documentation of ascitic fluid results require improvement. Increasing awareness on the importance of these sections in minimal standards of care amongst doctors and nurses will be the key factor in ensuring continual improvement in the quality of patient care.

\section{PTH-55 NEXT AVAILABLE PLEASE: REAL WORLD WAITING TIMES AND AVAILABILITY OF ENDOSCOPY FOR LOWER GASTROINTESTINAL BLEEDING}

${ }^{1}$ Mike Davies, ${ }^{2}$ Matthew Dixon*, ${ }^{3}$ Tristan Townsend, ${ }^{4}$ Violeta Razanskaite, ${ }^{2}$ James Morgan, ${ }^{5}$ Daniyal Baig, ${ }^{6}$ James Fox, ${ }^{6}$ Vinay Kumar, ${ }^{4}$ Nada Elamin, ${ }^{4}$ lannis Papmargaritas, ${ }^{3}$ Doug Penman, ${ }^{3}$ Thomas Conley, ${ }^{3}$ Joseph Fiske, ${ }^{2}$ Kieran Walker, ${ }^{7}$ Waqas Gaba, ${ }^{3}$ Sreedhar Subramanian, ${ }^{3}$ Philip J Smith. ${ }^{1}$ Countess of Chester Hospital, Chester, UK ${ }^{2}$ Arrowe Park Hospital, UK; ${ }^{3}$ Royal Liverpool University Hospital, Liverpool, UK; ${ }^{4}$ Aintree University Hospital, Liverpool, UK; ${ }^{5}$ Whiston Hospital, UK; ${ }^{6}$ Macclesfield District General Hospital, Macclesfield, UK; ' Southport Hospital, Southport, UK

\subsection{6/gutjnl-2021-BSG.370}

Introduction Current British Society of Gastroenterology (BSG) guidelines recommend all patients admitted to hospital with lower gastrointestinal bleeding (LGIB) undergo an inpatient colonoscopy on the next available list as part of providing a 7-day endoscopy service. The real-world availability of a 7-day service and adherence to this guidance is unknown.

Methods Patients aged $\geq 16$ years admitted with LGIB to 7 hospital trusts from June 1st-Aug 31st 2019 were included. Data on presentation, endoscopy, time to procedure and outcomes were recorded. We established data from participating Trusts on their current endoscopy services in relation to BSG guidance.

Results 407 patients across 7 NHS Trusts presenting with LGIB were included. Mean age was 59.8 (17-96), with a mean Oakland score of 14.5 (SD 6.78). 14.7\% received a LGI endoscopic investigation during admission (3.4\% colonoscopy, $11.8 \%$ flexible sigmoidoscopy), with $25.7 \%$ of admitted patients undergoing their LGI endoscopy as an outpatient. Median time from admission to inpatient flexible sigmoidoscopy and colonoscopy was 2.5 days (58 hours) and $>3$ days (80 hours) respectively. 43\% (3/7) of Trusts provided daily inpatient endoscopy lists able to accommodate inpatient colonoscopies, with colonoscopies being ad hoc/no regular slots in a further $43 \%$ of Trusts. At weekends, 86\% (6/7) of hospitals provided endoscopy lists, however of these, $83 \%$ stated colonoscopies were not routinely performed at weekends.

Conclusions Current real-world practice is not in keeping with BSG guidelines. The majority of patients admitted with LGIB do not undergo inpatient LGI endoscopy (colonoscopy or flexible sigmoidoscopy) and the waiting time for a 'next available' slot can be several days. LGI endoscopic assessment for LGIB is more commonly performed as an outpatient. Most Trusts do not currently provide a 7-day endoscopy service, the majority with no regular weekend or weekday colonoscopy slots for patients with LGIB.

\section{PTH-56 ASSESSMENT OF HIGH-RISK RECTAL POLYPS : LESSONS FROM A SPECIALIST MDT}

Douglas Penman*, Paul O'toole. Liverpool University Foundation Trust, Liverpool, UK
Introduction Recent guidelines have emphasised the importance of avoiding piecemeal resection of polyps that might contain malignancy. Moreover, superficial T1 cancers can be cured by local resection, avoiding the need for surgery. Evaluation of significant polyps and early colorectal cancers (SPECC) to determine the depth of invasion is of upmost importance. Assessment is difficult and no gold standard exists. In this study we evaluate the performance of our specialist MDT and, where sub-optimal assessment resulted in a poor outcome, we attempt to derive lessons that might be learnt.

Methods Referrals to the Specialist Early Rectal Cancer MDT were reviewed from Jan 2014 - Dec 2019. At the time of referral, they were categorised into: Confirmed early rectal cancers, referred for a primary treatment decision; inadvertently excised cancers, referred for possible adjuvant treatment and SPECC lesions, referred for a primary treatment decision. Each SPECC lesion was retrospectively split into 4 categories based on the MDT's risk of submucosal invasion: Low (category A), suitable for piecemeal resection; intermediate (B), suitable for en-bloc resection; high (C), suitable for TEMS and very high (D), not suitable for local excision. Where not explicit, an expert consensus was reached. Each assessment modality was compared to the MDT recommendation and final histological staging.

Results 581 referrals were made, 69 didn't meet referral criteria and 5 patients didn't engage or died before assessment. 57 patients were referred to consider adjuvant treatment for an inadvertently resected cancer. Of the remaining 450, 100 lesions were judged to be definite cancers and the remaining 350 were classed as SPECC lesions. Patients with SPECC lesions had a median age of 70 years, 2:1 male. The median and mean size of lesions was $38 \mathrm{~mm}$ and $42.8 \mathrm{~mm}$ respectively, range $10-150 \mathrm{~mm}$. Just over $20 \%$ of the lesions referred were larger than $60 \mathrm{~mm}$. Category A contained 174 lesions with a 4.8\% cancer rate (CR). Cat B: 108 lesions, 8.3\% CR. Cat C: 59 lesions, 54.2\% CR. Cat D 9 lesions, 57.1\% CR. 20\% of the lesions reported as mucosal (or not seen) on MRI were malignant compared with $10 \%$ on EUS. 8 malignant lesions were assigned to the low-risk category and subjected to pEMR. Adequate EUS staging was only available in 4 of these lesions. 4 out of the 11 lesions referred for surgery were benign.

Conclusion $83 \%$ of malignant polyps were triaged to an enbloc excision. The risk of malignancy reinforces the importance of evaluating SPECC lesions carefully. Exactly how the depth of invasion was predicted by the MDT is difficult to determine retrospectively. However, endoscopic evaluation, pre-resection dysplasia grade and EUS findings carry the greatest sway. MRI evaluation appears to be poor in predicting the depth of invasion for tumours $<\mathrm{T} 2$. II + IIc morphology and high grade dysplasia were the two greatest predictors of malignancy, $44 \%$ and $35 \%$ respectively.

\section{PTH-57 COLLABORATION BETWEEN ACUTE MEDICAL AND HEPATOLOGY TEAM SIGNIFICANTLY IMPROVES OUTCOMES OF PATIENTS WITH DECOMPENSATED CIRRHOSIS}

Saqib Mumtaz*, Maria Tan, Andrea Adjetey, Chris Corbett, lan Perry, Azeez Olajide, James Owen. Royal Wolverhampton Hospital, NHS Trust, Wolverhampton, UK 